\title{
Telugu Language
}

National Cancer Institute

\section{Source}

National Cancer Institute. Telugu Language. NCI Thesaurus. Code C154153.

A South-central Dravidian language native to India, where it is the primary language in the states of Andhra Pradesh, Telang ana, and the union territory of Puducherry. 